\title{
Evaluación de la globulina transportadora de hormonas esteroidales (SHBG) durante el embarazo como factor predictor de pre-eclampsia y restricción del crecimiento intrauterino
}

ENRIQUE VALDÉS R. ${ }^{1}$, KARINA LATTES A. ${ }^{1}$, HERNÁN MUÑOZ S. ${ }^{1}$, MIGUEL ÁNGEL CUMSILLE²

\section{Sex-hormone binding globulin (SHBG) levels during pregnancy as predictors for pre-eclampsia and fetal growth restriction}

Background: Sex-Hormone Binding Globulin (SHBG) may be associated to Pre-eclampsia (PE) and Fetal Growth Restriction (RCIU). Aim: To determine if maternal serum SHBG concentrations during the first and second trimesters are predictive biomarkers of Pre-eclampsia and RCIU. Patients and Methods: Prospective cohort study carried out in the Fetal Medicine Unit, Universidad de Chile Clinical Hospital between January, 2005 and December, 2006. Blood samples were obtained from unselected pregnant women during routine 11-14 week and 22-25 week ultrasound examinations, conforming two different study groups. Posteriorly, serum SHBG concentrations were determined in women who developed Pre-eclampsia, $R C I U$ and their respective controls. Results: Fifty five patients were included in the 11-14 weeks group. Nine women that developed PE, 10 that developed RCIU and 36 controls were selected from this group. There were no significant differences in SHBG levels between patients with PE, RCIU or controls (324.7 (26.6), 336.8 (33.9) and 377.5 (24.3) nmol/L, respectively). Fifty four women were included in the 22-25 weeks group. Eight women who developed Pre-eclampsia, 15 who developed RCIU and 31 controls were selected. Again, there were no significant differences in SHBG levels between patients with PE, RCIU or controls (345.5 (151.1), 383.8 (143.4) and $345.5 \mathrm{nmol} / \mathrm{l}$ (151.1), respectively). Conclusions: Maternal SHBG serum levels did not predict subsequent development of Pre-eclampsia and RCIU.

(Rev Med Chile 2012; 140: 589-594).

Key words: Fetal development; Pre-eclampsia; Sex hormone-binding globulin.

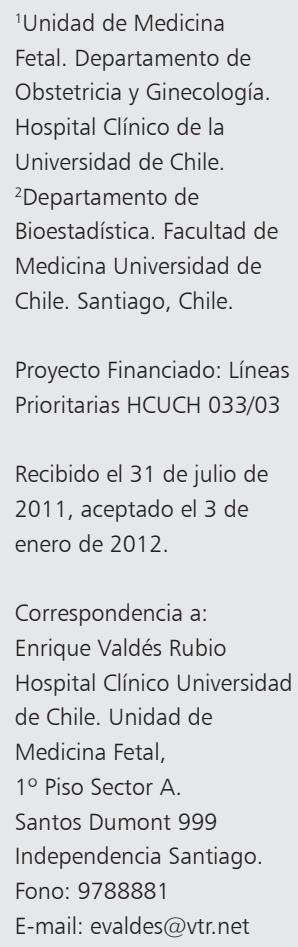

\section{Introducción}

a pre-eclampsia (PE) y la restricción del crecimiento intrauterino (RCIU) son pato$\checkmark$ logías de alto riesgo perinatal que, a pesar de presentar una etiopatogenia multifactorial y parcialmente conocida comparten una base anatómica en común, una placentación anómala ${ }^{1-3}$.

La PE es una patología partícipe del síndrome hipertensivo del embarazo (SHE), caracterizada por hipertensión arterial y proteinuria que afecta a 4-7\% de la población gestante y que representa un alto riesgo de morbi-mortalidad materno-perinatal. En la actualidad no se cuenta con métodos que permitan su detección temprana, como tampoco de intervención terapéutica alguna que permita evitar o retardar la aparición de esta enfermedad ${ }^{3-7}$.

La RCIU se caracteriza como un feto de menor 
peso a lo esperado en relación a su edad gestacional y sexo definiéndose como aquel que crece por debajo del percentil 10 de una curva de crecimiento representativa de la población ${ }^{8}$. Esta patología es considerada de alto riesgo ya que es responsable de prematuridad y de ocasionar complicaciones en el desarrollo psicomotor en la infancia y alteraciones metabólicas y cardiovasculares en la vida adulta ${ }^{8}$.

La PE y RCIU son las patologías que concentran la mayor proporción de morbimortalidad al binomio madre-hijo. Fisiopatológicamente, una de las teorías que explicaría la génesis de estas patologías se basa en una placentación anómala, cuyo origen anatomopatológico sería una alteración de la migración fisiológica del trofoblasto extra-vellositario al interior de las arterias espirales maternas durante la primera mitad del embarazo que impediría la transformación de un territorio originalmente de alta resistencia y baja capacitancia en uno de baja resistencia y alta capacitancia, efecto agravado por el desarrollo de trombosis de los vasos conformantes de la placenta que terminaría provocando la insuficiencia de este órgano ${ }^{1-3}$.

Se han propuestos modelos con el fin de predecir la PE y RCIU en edades gestacionales tempranas, pero por una parte estos presentan un bajo valor predictivo y su implementación es de alto costo económico y, por otra, no aportan intervención profiláctica alguna que permita la prevención o el retraso de la aparición de estas patologías $5,9,10$.

Actualmente, existen evidencias contradictorias que sugieren que la alteración en la concentración plasmática de una serie de biomarcadores asociados a la insulinorresistencia (IR) (SHBG, adiponectina, leptina), en diferentes etapas del embarazo, estarían relacionadas con patologías que afectan al binomio madre-hijo en cuya etiopatogenia se encuentra presente la disfunción endotelial. Esta disfunción favorecería condiciones protrombóticas placentarias y sistémicas y/o condiciones metabólico-hormonales que se relacionarían a una placentación anómala como la observada en PE y RCIU, no obteniéndose aún resultados definitivos ${ }^{11-20}$.

La SHBG es una glicoproteína sintetizada por el hígado, que liga estrógenos y testosterona circulantes. La producción de ésta es inhibida por insulina, aun en situaciones de aumento de niveles de estrógeno, observación especialmente válida durante el embarazo en donde las concentra- ciones de estrógenos, SHBG e insulina aumentan dramáticamente ${ }^{16,19}$. Estos niveles reducidos de SHBG son considerados como un marcador de hiperinsulinemia y de $\mathrm{IR}^{21,22}$, independientemente del índice de masa corporal y de su distribución grasa. En el embarazo normal la concentración sérica de SHBG aumenta linealmente durante el primer y segundo trimestre, alcanzando su máximo a las 24 semanas (4-6 veces los niveles de mujeres no embarazadas $)^{14}$.

El presente estudio tiene como objetivo determinar si la concentración plasmática materna de SHBG en el primer y segundo trimestre del embarazo es un biomarcador predictor de PE, RCIU o ambos.

\section{Metodología}

\section{Diseño del protocolo}

Se realizó un estudio anidado caso-control en 1094 mujeres embarazadas que iniciaron su control prenatal en el Servicio de Maternidad del Hospital Clínico de Universidad de Chile entre enero de 2005 y diciembre de 2006 y que posteriormente tuvieron su parto.

Se incluyeron gestantes cursando embarazo único cuyo parto se presentó después de las 20 semanas de gestación y quedaron excluidas aquellas que presentaron historia de diabetes mellitus pregestacional, enfermedad tiroídea, hepática, nefropatía crónica o hipertensión arterial crónica (definida como presiones arteriales mayores o iguales a 140/90 $\mathrm{mmHg}$, que estén en tratamiento actual con antihipertensivos o necesiten de su uso antes de las 20 semanas de gestación).

Para el análisis final sólo fueron incluidas aquellas gestantes que desarrollaron una de ambas patologías, ya que por su etiopatogenia parcialmente común, una proporción de estas pacientes pueden presentarlas en concomitancia.

Después de contar con el consentimiento informado aprobado por el Comité de Ética del Hospital Clínico Universidad de Chile, se procedió a tomar una muestra sanguínea de $5 \mathrm{ml}$ a todas las pacientes embarazadas entre las 11-14 y 22-24 semanas de gestación que ingresaron a control a la Unidad de Medicina Fetal del Servicio de Maternidad del Hospital Clínico de la Universidad de Chile durante el mismo período. Estas fueron congeladas a $-80^{\circ} \mathrm{C}$ para futuros análisis de la concentración plasmática de SHBG posteriores 
al parto por método de ELISA. Datos biomédicos tanto de la evolución del embarazo, parto y del recién nacido se consignaron en base de datos para cada paciente en estudio. Una vez conocidos los resultados perinatales al término del embarazo, se procedió a seleccionar, descongelar y evaluar las concentraciones plasmáticas de SHBG de aquellas pacientes que desarrollaron PE o RCIU y sus respectivos dos controles sin patología en los dos periodos estudiados (11-14 y 22-25 semanas), teniendo presente que ambos grupos son conformados por diferentes pacientes.

Para este estudio se definió como preeclampsia al cuadro clínico caracterizado por hipertensión asociada a proteinuria después de las 20 semanas de gestación que cumpla con los siguientes criterios: cifras tensionales mayores o iguales a 140/90 $\mathrm{mmHg}$ en dos tomas separadas por 6 horas de observación en reposo o que presente sólo una cifra de 160/100 mmHg ó mayor, asociado a proteinuria cuantitativa mayor a 300 mg en orina de 24 horas, en ausencia de infección del tracto urinario ${ }^{23}$.

Por otra parte, se definió como RCIU aquel feto cuyo peso está por debajo del percentil 10 de una curva de crecimiento representativa de la población chilena en relación a su edad gestacional y sexo ${ }^{24}$, diagnóstico realizado después de las 20 semanas. Se consignó la variable peso del recién nacido (PRN) para ratificar el diagnóstico prenatal de RCIU y para su análisis en relación a las otras variables en estudio.

\section{Técnicas}

\section{1) Evaluación nutricional:}

Al momento de la toma de la muestra se medió el peso $(\mathrm{kg})$, utilizando una balanza de palanca (Detecto) con una precisión de $0,1 \mathrm{~kg}$, y la estatura (m) con un cartabón adosado a la balanza. Con estos datos se calculó el índice de masa corporal $\left(\mathrm{IMC}=\mathrm{kg} / \mathrm{m}^{2}\right)$ el cual se clasificó de la siguiente manera: (IMC $<25=$ normal; IMC $\geq 25$ sobrepeso; IMC $\geq 30$ obesidad; IMC $\geq 40$ obesidad mórbida).

\section{2) Parámetros bioquímicos de IR:}

SHBG: Análisis enzimoinmunométrico por quimioluminiscencia para equipo automatizado INMUNOLITE (DPC representado en Chile por Clinitest).

\section{3) Métodos estadísticos}

Se empleó un análisis de regresión simple para las variables cuantitativas como; edad materna, IMC, PRN. Debido al bajo número muestral, para el análisis de las variables cualitativas: Primigesta, Tabaco y Alcohol, se aplicó prueba exacta de Fisher.

Para la comparación de los valores de concentración plasmática de SHBG entre los grupos de PE, RCIU y Control, se realizó la prueba de normalidad de Shapiro-Wilk. Posteriormente y según correspondió, se aplicó ANOVA a una sola vía (one way), con análisis post hoc la prueba de Scheffe para comparaciones múltiples o la prueba de Kruskall Wallis. Para todas las mediciones se consideró un valor de p de 5\%.

\section{Resultados}

Debido a la exclusión del estudio de aquellas pacientes que compartían ambas patologías, hecho que es frecuente, ya que ambas enfermedades compartirían etiopatogenia en común, los grupos analizados quedaron conformados de la siguiente manera:

1) El grupo estudiado entre las 11-14 semanas estuvo constituido por 55 pacientes de las cuales se seleccionaron 9 pacientes que desarrollaron PE, 10 que desarrollaron RCIU y 36 controles. $\mathrm{Al}$ analizar los datos demográficos y biomédicos mediante regresión simple se encontró que aquellas gestantes que desarrollaron PE presentaron IMC significativamente mayores [mediana 29,2 $\mathrm{kg} / \mathrm{m}^{2}(5,6)$ vs $23,72 \mathrm{~kg} / \mathrm{m}^{2}(3,4)$ p 0,01$]$ y PRN significativamente menores que el grupo control [PRN $2.351 \mathrm{~g}(1.395)$ vs $3,489 \mathrm{~g}(425,4)$ p 0,0001 ] y encontrándose mayor incidencia de PE y RCIU en primigestas [ $44 \%$ vs $33 \%$ p 0,03 y $70 \%$ v/s $33 \%$ p 0,01, respectivamente] Tabla 1 . Los resultados obtenidos para la concentración sérica de SHBG entre las 11-14 semanas, sus respectivas medianas, desviaciones estándar y significancias se muestran en la Tabla 2. No se encontraron diferencias significativas en la concentraciones séricas de SHBG entre aquellas embarazadas que desarrollaron $\mathrm{PE}$, RCIU y controles [medianas en el grupo PE 324,7 nmol/l $(26,6)$ p 0,43 ; RCIU 336,8 nmol/l $(33,9)$ p 0,56 y Control $377,5 \mathrm{mnol} / \mathrm{l}(24,3)]$.

2) El grupo estudiado entre las $22-25$ semanas estuvo constituido por 54 pacientes de las cuales se seleccionaron 8 pacientes que desarrollaron $\mathrm{PE}, 15$ que desarrollaron RCIU y 31 controles. Al estudiar 
los datos demográficos y biomédicos mediante regresión simple se encontró mayor incidencia de RCIU en el grupo de primigestas [66\% vs 33\%] y un PRN significativamente menor en los hijos cuyas madres desarrollaron PE [ $2.758 \mathrm{~g}$ vs $3.339 \mathrm{~g} \mathrm{p}$ $0,001]$ Tabla 3. Asimismo, en este grupo analizado no se encontraron diferencias significativas en la concentraciones séricas de SHBG entre aquellas embarazadas que desarrollaron PE, RCIU y controles [medianas en el grupo PE $345,5 \mathrm{nmol} / 1$ $(151,1)$ P 0,78, RCIU 383,8 nmol/l(143,4) p 0,36 Control 345,5 nmol/l $(151,1)$. Tabla 4.

Tabla 1. Características demográficas maternas y neonatales 11-14 semanas

\begin{tabular}{|c|c|c|c|c|c|}
\hline & RCIU (10) & PE (9) & Controles (36) & & $\mathbf{p}$ \\
\hline $\begin{array}{c}\text { Edad, años } \\
\text { Mediana } \\
\text { Rango }\end{array}$ & $\begin{array}{r}28,1(6,7) \\
19,6-36,6\end{array}$ & $\begin{array}{r}29,5(5,1) \\
23,4-37,7\end{array}$ & $\begin{array}{c}29,9 \quad(6,2) \\
17,5-42,2\end{array}$ & $0,75^{*}$ & $0,81^{* *}$ \\
\hline Primigesta n (\%) & 7 (70) & $4(44,4)$ & $12(33,3)$ & 0,01 * & $0,03^{* *}$ \\
\hline Tabaco n (\%) & $2(5,5)$ & $0(0)$ & & & \\
\hline Alcohol n (\%) & $2(5,5)$ & $1(5,26)$ & & & \\
\hline $\mathrm{IMC}\left(\mathrm{kg} / \mathrm{m}^{2}\right)$ & $24,1(4,6)$ & $29,2(5,6)$ & $23,72(3,4)$ & $0,76^{*}$ & $0,01^{* *}$ \\
\hline PRN (Mediana) (g) & $2.758(92,1)$ & $2.351(1.395)$ & $3.489(425,4)$ & $0,0001 *$ & $0,0001 * *$ \\
\hline
\end{tabular}

Resultados de las características demográficas maternas y neonatales de casos y controles en grupo de gestantes en que se midió SHBG entre las 11 y 14 semanas.

- RCIU vs controles.

- PE vs controles.

Tabla 2. Características demográficas maternas y neonatales 22-25 semanas

\begin{tabular}{|c|c|c|c|c|c|}
\hline & RCIU (15) & PE (8) & Controles (31) & & \\
\hline $\begin{array}{c}\text { Edad, años } \\
\text { Mediana } \\
\text { Rango }\end{array}$ & $\begin{array}{r}27,6(5,0) \\
21,4-36,8\end{array}$ & $\begin{array}{r}29,2(3,8) \\
23,2-33,8\end{array}$ & $\begin{array}{r}30,8(5,8) \\
17,4-41,5\end{array}$ & $0,14^{*}$ & $0,51 * *$ \\
\hline Primigesta $\mathrm{n}(\%)$ & $10(66,6)$ & $3(37,5)$ & $12(33,3)$ & $0,02^{*}$ & $0,97^{* *}$ \\
\hline Tabaco n (\%) & $0(0)$ & $2(5,5)$ & $3(9,6)$ & & \\
\hline Alcohol n (\%) & $1(5,26)$ & $2(5,5)$ & & & \\
\hline PRN (Mediana) (g) & $2.382(637)$ & $2.758 \pm 32$ & $3.339(520)$ & 0,0001 * & 0,001 ** \\
\hline IMC $\left(\mathrm{kg} / \mathrm{m}^{2}\right)$ & $23,5(2,9)$ & $29,0(5,2)$ & $24,8(4,7)$ & $0,32^{* *}$ & $0,48^{* *}$ \\
\hline
\end{tabular}

Resultados de las características demográficas maternas y neonatales de casos y controles en grupo de gestantes en que se midió SHBG entre las 22-25 semanas.

- RCIU vs controles.

- PE vs controles.

Tabla 3. Concentraciones de SHBG (nmol/l) 11-14 semanas

\begin{tabular}{|lccc|}
\hline & n pctes & Mediana & p \\
\hline PE & $(9)$ & $324,7(26,6)$ & 0,43 \\
RCIU & $(10)$ & $336,8(33.9)$ & 0,56 \\
Control & $(36)$ & $377,5(24.3)$ & \\
\hline
\end{tabular}

Resultados de concentración plasmática de SHBG (nmol/l) tomadas en gestantes entre las 11 y 14 semanas en casos y controles.
Tabla 4. Concentraciones de SHBG (nmol/l) 22-25 semanas

\begin{tabular}{|lcccc|}
\hline & n pctes & Mediana & D/S & p \\
PE & $(8)$ & 345,5 & $(151,1)$ & 0,78 \\
RCIU & $(15)$ & 383.8 & $(143.4)$ & 0,36 \\
Control & $(31)$ & 345,5 & $(151,1)$ & \\
\hline
\end{tabular}

Resultados de concentración plasmática de SHBG (nmol/l) tomadas en gestantes entre las 22 y 25 semanas en casos y controles. 


\section{Discusión}

En el presente estudio, las concentraciones séricas maternas de SHBG durante el primer trimestre en las gestantes que desarrollaron PE y RCIU no difieren de las encontradas en las gestantes controles. Si bien es cierto, Wolf ${ }^{20,21}$ publicó uno de los primeros estudios prospectivos donde se relacionó la menor concentración de SHBG medida en el primer trimestre con el posterior desarrollo de PE, estudios metodológicamente bien diseñados no han confirmado esta asociación. Es así que Spencer no encontró diferencias significativas en la concentración de esta hormona durante el primer trimestre entre las pacientes que desarrollaron $\mathrm{PE}$, RCIU y los controles ${ }^{25}$, pesquisando solamente que menores concentraciones se relacionaban a una incidencia mayor de abortos tempranos y el reconocido mayor riesgo de desarrollar diabetes gestacional.

Coincidentemente, en el grupo estudiado entre las 22-25 semanas tampoco se encontró una diferencia significativa en las concentraciones séricas de SHBG en aquellas embarazadas que desarrollaron PE o RCIU y el grupo control, lo que difiere con lo publicado por Yu quien concluyó que aquellas gestantes que desarrollaban posteriormente PE presentaban valores significativamente menores de $\mathrm{SHBG}^{26}$.

Con relación a las características biomédicas estudiadas destaca el significativo menor PRN pesquisado en las pacientes que desarrollaron $\mathrm{PE}$ versus los controles, efecto ponderal esperado debido a la necesidad de interrupción del embarazo prematuramente por indicación médica según severidad de PE y su esperada asociación en algunos casos con RCIU.

Se ha relacionado el estado mórbido nutricional materno definido como a altos niveles de índice de masa corporal (IMC) pre y post concepcionales y/o el exagerado incremento de peso durante la gestación como un factor de riesgo en el desarrollo de $\mathrm{PE}^{27,28}$.

En este estudio el peso preconcepcional conjuntamente con el reconocido factor de riesgo de la nuliparidad, se relacionó con aparición de PE posterior en el grupo entre las 11-14 semanas, lo que se traduciría como factor de riesgo en la aparición de esta patología, variable independiente de la concentración de SHBG y que podría ser secundario a un factor inflamatorio, variable no estudiada en este proyecto. Con relación a esto último, es reconocido el rol de la disfunción endotelial en la etiología de la PE y si bien es cierto aún no se conoce su causa, se piensa que es secundaria a una respuesta inflamatoria sistémica materna. Por otra parte, la disfunción endotelial está relacionada a la ateroesclerosis, enfermedad inflamatoria que tiene una serie de características metabólicas similares a la PE. Además en placentas complicadas con PE muestran aterosis de las arterias espirales las que serían responsables de trombosis en estos vasos que estarían en estrecha relación a la etiopatogenia de esta enfermedad. Las evidencias que apoyan la relación de un factor inflamatorio e IMC en la génesis de la PE son contradictorias ya que si bien es cierto Wolf encontró un aumento significativo de proteína $\mathrm{C}$ reactiva (marcador de inflamación sistémica) en pacientes con IMC mayores que posteriormente desarrollaban $\mathrm{PE}^{28}$, el mismo autor determinó que la IR y no el factor inflamatorio sería un factor de riesgo independiente en el desarrollo de síndrome hipertensivo de embarazo ${ }^{20}$. Esta conclusión no es necesariamente extrapolable a PE, ya que cada vez se tiene más claro que esta última tiene una etiopatogenia y un comportamiento fisiopatológico diferente del síndrome hipertensivo de embarazo.

Recientemente, $\mathrm{y}$ a pesar que se ha intentado relacionar las concentraciones séricas de SHBG entre las11-14 y 22-25 semanas como biomarcador en la predicción temprana de PE y RCIU, este estudio no apoyaría dicha relación con estas últimas ${ }^{19,26}$.

En resumen, el presente estudio con un número reducido de pacientes, no logró evidenciar una relación entre los niveles plasmáticos maternos de SHBG en el primer y segundo trimestre y el posterior desarrollo de PE y RCIU. Estableciéndose como factor de riesgo de desarrollar PE la primiparidad y grados mayores de IMC pregestacional. Dado el reducido tamaño muestral, estos hallazgos deben ser ratificados con estudios posteriores con un mayor número de gestantes.

\section{Referencias}

1. Khong TY, De Wolf F, Robertson WB, Brosens I. Inadequate maternal vascular response to placentation in pregnancies complicated by preeclampsia and by small for gestational age infants. Br J Obstet Gynaecol 1986; 93: 1049-59. 
2. Roberts JM, Redman CWG. Pre-eclampsia: More than pregnancy induced hypertension. Lancet 1993; 341: 1447-54.

3. Campbell S, Pearce JM, Hackett G, Cohen-Overbeek T, Hernández C. Cualitative asssessment of uteroplacental blood flow: early screening test for high-risk pregnancies. Obstet Gynecol 1986; 68: 649-53.

4. Sibai BM. Prevention of preeclampsia: a big disappointment. Am J Obstet Gynecol 1998; 179: 1275-8.

5. Arngrimsson R, Björnsson S, Geirsson RT, Björnsson H, Walker JJ, Snaedal G. Genetic and familial predisposition to eclampsia and preeclampsia in a definited population. Br J Obstet Gynecol 1990; 97: 762-9.

6. Robillard PY, Hulsey TC, Perianin J, Janky E, Miri EH, Papiernik E. Association of pregnancy induced hypertention with duration of sexual cohabitation before conception. Lancet 1994; 344: 973-5.

7. Redman CW, Saks GP, Sargent IL. Preeclampsia and excessive maternal inflammatory responsive to pregnancy. Am J Obstet Gynecol 1999; 180: 499-506.

8. Barker DJP. Programming the baby. In mothers, babies and disease in later life. BMJ Publishing Group, London pp. 14-36.

9. Campbell S, Pearce JM, Hackett G, Cohen-Overbeek T, Hernández C. Cualitative asssessment of uteroplacental blood flow: early screening test for high-risk pregnancies. Obstet Gynecol 1986; 68 (5): 649-53.

10. Pilalis A, Souka AP, Antsaklis P, Daskalakis G, Papantoniou N, Mesogitis S, et al. Screening for pre-eclampsia and fetal growth restriction by uterine artery Doppler and PAPP-A at 11-14 weeks' gestation. Ultrasound Obstet Gynecol 2007; 29 (2): 135-40.

11. Ciaraldi TP, Kettel M, El-Roeiy A, Madar Z, Reichart D, Yen SCC, et al. Mechanisms of cellular insulin resistance in human pregnancy. Am J Obstet Gynecol 1994; 170 (2): 635-41.

12. Timar O, Sestier F, Levy E. Metabolic Síndrome X: a review. Can J Cardiol 2000; 16: 779-89.

13. Kirwan JP, Huston-Presley L, Kalhan SC, Catalano PM. Clinically useful estimates of insulin sensitivity during pregnancy: validation studies in women with normal glucose tolerance and gestational diabetes mellitus. Diabetes Care 2001; 24 (9): 1602-07.

14. Catalano PM, Tyzbir ED, Roman NM, Amini SM, Simus EAH. Longitudinal changes insulin release and insulin resistance in nonobese pregnant women. Am J Obst Gynec 1991; 165: 1667-72.

15. Barbieri RL. Endocrine disorders in pregnancy. 1999. Eds Reproducitve endocrinology. Philadelphia. Pa: WB Saunders Co.
16. Solomon CG, Seely EW. Hypertension in pregnancy: a manifestation of the insulin resistance syndrome? Hypertension 2001; 37: 232-9.

17. Sir-Petermann T, Hitchsfeld C, Maliqueo M, Codner E, Echiburú B, Gacitúa R, et al. Birth weight in offspring of mothers with polycyctic ovarian syndrome. Hum Reprod 2005; 20 (8): 2122-6.

18. Sir-Petermann T, Maliqueo M, Angel B, Lara HE, PérezBravo F, Recabarren SE. Maternal serum androgens in pregnant women with polycyctic ovarian syndrome: possible implications in prenatal androgenization Hum Reprod 2002; 17: 2573-9.

19. Wolf M, Sandler L, Muñoz K, Hsu K, Ecker JL, Thadhani R. First trimestre insulin resistance and subsequent preeclampsia: a prospective study. The J of Clin Endocr \& Metab 2002; 87(4): 1563-8.

20. Wolf M, Sander L, Jiménez-Kimble R, Shah A, Ecker J, Thadhani R. Insuline resistan but not inflammation is not associated with gestational hypertension. Hypertensio 2002; 40: 886-91.

21. Plimate SR, Matej LA, Jones RE Plimate SR, Matej LA, Jones RE. Inhibition of sex hormone-binding globulin production in the human hepatoma cell line by insulin and prolactin. J Clin Endocrinol Metab 1988; 67: 460-4.

22. Thadani R, Stampfer MJ, Hunter DJ, Manson JE, Solomon CG, Curhan GC. High body mass index and hypercholesterolemia: risk of hypertensive disorders of pregnancy. Obstet Gynecol 1999; 94: 543-50.

23. Brown MA, Lindheimer MD, de Swiet M, van Assche A, Moutquin JM. The classification and diagnosis of the hypertensive disorders of pregnancy: statement from the International Society for the Study of Hypertension in Pregnancy (ISSHP). Hypertens Pregnancy 2001; 20: ix-xiv.

24. Juez G, Lucero E, Ventura-Junca P, González H, Tapia JL, Winter A. [Intrauterine growth in Chilean middle class newborn infants]. Rev Chil Pediatr 1989; 60: 198-202.

25. Spencer K, Yu CK, Rembouskos G, Binda R, Nicolaides $\mathrm{KH}$. First trimester sex hormone-binding globulin and subsequent of preeclampsia or other adverse pregnancy outcomes. Hypertension Pregnancy, 2005;24(3):303-11.

26. Yu CK, Ppageorghiou AT, Binda R, Spencer K, Nicolaides $\mathrm{KH}$. Second-trimester sex hormone-binding globuling and subsequent development of pre-eclmpsia. J Matern Fetal Neonatal Med 2004; 16 (3): 158-62.

27. The ESHRE Capri Workshop Group. Human Reproduction Update 2006; 12: 193-207.

28. Wolf M, Kettyle E, Sandler L, Ecker J, Roberts J, Thadhani. Obesity and preeclampsia: The potential rol of inflammation. Obstet Gynecol 2001; 98: 757-62. 